\title{
La autonomía del centro escolar público, clave para el desarrollo del liderazgo directivo
}

\author{
Rafael González Martín ${ }^{1}$
}

Recibido: 10-07-2018

Aceptado: 04-09-2018

\section{RESUMEN}

El presente trabajo quiere resaltar la importancia del liderazgo educativo, como el camino por el que se debe de avanzar para conseguir esa escuela pública de calidad, tal como demanda la sociedad actualmente. Pero también, se pretende dejar claro en este trabajo que, la responsabilidad de esa calidad educativa que se demanda, dependerá en gran medida del propio centro, el cual, debe de estar cargado de cierta cota de autonomía que le permita poder desarrollar sus propios proyectos, adecuándolos a sus contextos, con el fin también, de pedir responsabilidades al liderazgo directivo de los resultados obtenidos.

Palabras clave: centros educativos, liderazgo directivo, calidad educativa.

1. Profesor en el Colegio María Auxiliadora. Salesianas Écija. Doctor por la Universidad de Murcia - Instituto Teológico de Murcia OFM en el programa de Teología. Mis líneas de investigación, están centrada en las áreas de la Teología y las Ciencias de la Educación. En el área de la Ciencias de la Educación, en la intervención y evaluación educativas sobre el análisis de necesidades, el diseño y aplicación de programas de intervención, así como la evaluación de programas, servicios y resultados educativos.

Bogotá - Colombia.

Correo: fr.gonzalezm@hotmail.com

ORCID: https://orcid.org/0000-0002-6114-4089

https://dialnet.unirioja.es/servlet/autor? codigo=3918361 


\title{
The autonomy of the public school center, key to the development of the leadership
}

\begin{abstract}
The present work wants to highlight the importance of educational leadership, as the way in which public schools must move forward to obtain the quality demanded by the current society. But also, it is important to clarify in this work that the responsibility for that demanded educational quality will depend on the center itself, which must be charged with a level of autonomy that allows each center to develop their own projects, adapting them to their contexts, in order also to be able to ask for responsibilities to the director leadership for the results obtained.
\end{abstract}

Keywords: educational centers, director leadership, educational quality.

\section{L'autonomie du centre scolaire public, clé du développement du leadership leadership}

\section{RÉSUMÉ}

Dans ce document se démarque l'importance du leadership éducatif comme le chemin par lequel on doit avancer pour que les écoles publiques seraient de la qualité tel que la société actuelle exige. Mais on prétend aussi clarifier dans cette recherche que la responsabilité de cette qualité éducative exigée, dépendra en chaque centre éducatif, lesquels doivent avoir l'autonomie qui soit nécessaire pour le développement leurs propres projets et les adapter au leurs contexte, afin de demander au leadership directif des résultats obtenus.

Des mots clés: centre éducatif, leadership direct, qualité éducative. 


\section{A autonomia do centro escolar público, chave para o desenvolvimento da liderança}

\section{RESUMO}

O presente trabalho pretende ressaltar a importância da liderança educacional, como forma de avançar para a obtenção de uma escola pública de qualidade, como a sociedade exige atualmente. Mas também, pretende-se deixar claro neste trabalho que a responsabilidade pela qualidade educacional exigida dependerá, em grande parte, do próprio centro, que deve estar carregado de um certo nível de autonomia que permita a cada centro desenvolver seus próprios projetos, adaptando-os aos seus contextos, para também poder solicitar à liderança liderança os resultados obtidos.

Palavras-chave: centros educativos, liderança gerencial, qualidade educacional.

\section{Introducción}

La dirección escolar en España, está en un proceso de profunda transformación, para poder satisfacer las necesidades que la sociedad demanda al sistema educativo. Un proceso que va oscilando desde, un modelo directivo burocrático-administrativo, para pasar a un liderazgo transformador de dirección pedagógica para el aprendizaje, como un factor de primer orden en los logros académicos de los alumnos, así como de los resultados del centro escolar (Pérez, 2013). De aquí que, hoy en día, la mejora de los resultados en un centro escolar se está convirtiendo en una prioridad permanente dentro del sistema educativo, llegándose a relacionar el éxito o fracaso del centro a la dirección de él.

El autor de este trabajo, quiere destacar que, los resultados obtenidos por un centro escolar público no pueden de manera exclusiva ser responsabilidad de la dirección. Si no que estos, están influenciados a su vez, por una serie de variables contextuales que surgen en dos niveles. Un 
nivel externo, que viene enmarcado en un primer lugar por el contexto socio-económico y cultural en el que se encuentra el centro (Langer, 2016) y, lógicamente también por todos los factores que condicionan el hacer profesional de la dirección (política educativa, normativa reguladora, autonomía, recursos, tipo de escuela...); y un nivel interno que condiciona al líder directivo en el centro escolar, como el profesorado y los órganos de gobierno (de la Calle et al., 2014). Pues, no podemos negar, como hemos dicho anteriormente, hoy en día, la dirección de un centro público, cuenta con poco poder y poca autonomía para ejercer un liderazgo pedagógico, transformacional y estratégico, al verse supeditado a la poca capacidad de maniobra en la gestión de personal, de tiempo y recursos.

En el presente trabajo se propone, como estratégica de liderazgo directivo, un liderazgo distributivo o compartido, como medio y marco que permita satisfacer las necesidades educativas de los centros escolares afectados principalmente por una plantilla inestable, con el valor añadido que, todo esto conlleva, a la hora de formar un equipo directivo respaldado por los demás órganos de gobierno y que, a su vez, lógicamente permita llevar a buen término el Proyecto Educativo de Centro. Ante circunstancias como estás presentadas en este trabajo, el compromiso y la responsabilidad de todo el profesorado, incluida la comunidad, debe ser la de contribuir activamente todos sus miembros a dinamizar, apoyar y animar, como arma fundamental para que su centro supere los retos y dificultades a los que tiene que enfrentarse (Viejo, Cabezas \& Martínez, 2103). Con ésta estrategia, se presenta un liderazgo que emergerá, desde un grupo de personas que trabajan juntas en torno a unos objetivos comunes; como bien señala de Alma Harris, Kenneth Leithwood, Christopher Day, Pam Sammons y David Hopkins (Bolívar, 2010).

Se presenta este trabajo, dentro de una estrategia de liderazgo distributivo, como un planteamiento innovador $y$, bajo el pleno convencimiento de que es el marco idóneo para resolver y configurar un liderazgo en aquellos centros, en los cuales es difícil su definición, como consecuencia de su inestable estructura organizativa. Así también, queda recogido en los actuales estudios e investigaciones, que nos presentan las diversas literaturas que tenemos al respecto (Bolivar, 2010). 


\section{Proyecto de Dirección de Centro}

\section{Contexto socio-económico}

El centro se encuentra situado en una pedanía, que dista a unos 3,5 km de la localidad principal a la que pertenece administrativamente. Junto con los 1000 habitantes pedáneos, constituye un Ayuntamiento de menos de 5.000 habitantes, con pocos recursos económicos y con la circunstancia de una duplicidad de núcleos poblacionales, lo que le obliga a prestar los mismos servicios en las dos localidades: salón de usos múltiples, guardería, piscina pública, polideportivo, etc., con la consiguiente duplicidad en los costes económicos. A pesar de estas circunstancias, el Ayuntamiento hace grandes esfuerzos por conseguir una dotación sociocultural adecuada para ambos núcleos poblacionales, considerándose la infraestructura de este tipo en la pedanía como adecuada.

La población masculina trabaja principalmente en la agricultura y en el sector de la construcción, siendo el sector terciario poco significativo, aunque en los últimos años va tomando cierta importancia. Las mujeres son principalmente trabajadoras agrícolas estacionarias (campañas de la aceituna, cebollas, etc.). La presencia de varias empresas auxiliares del sector de la construcción, especialmente dedicada a la tabiquería prefabricada, en la localidad ha originado un gran bienestar económico y de empleo, con un bajo índice de paro en la misma, durante los años de la gran bonanza inmobiliaria. Durante estos años de crecimiento inmobiliario, el varón se ve obligado a permanecer fuera de casa durante toda la semana para realizar su actividad laboral en la costa principalmente. Recayendo durante la semana, la responsabilidad familiar estrictamente en la figura de la madre. El nivel económico de las familias durante estos años de bonanza económica es muy aceptable, dándose pocos casos de familias con necesidades económicas graves. Situación que en la actualidad es todo lo contrario (Ferreyra, 2014).

El índice de natalidad es muy bajo, siendo muy considerable en los últimos años, y al ser prácticamente nula la llegada de inmigrantes de otras nacionalidades, la población infantil va disminuyendo y la adulta envejeciendo.

Al ser un núcleo reducido, las posibilidades culturales son bajas. La cercanía de dos localidades a $15 \mathrm{Kms}$. alivia en parte esta carencia. El 
centro escolar, podemos decir, se constituye así como la institución cultural más importante de la localidad.

\section{Características internas del Centro}

La primera característica particular del Centro es el hecho de ser un SemiD, es decir, centro que además de Infantil y Primaria, imparte el primer ciclo de la ESO. Esta particularidad hace que el centro cuente actualmente con una ratio de unos 20 alumnos/as por clase, dado que en las dos unidades de ESO el número de alumno-as es superior a 25 por la cantidad de repetidores que se acumulan en este nivel. En total, la configuración actual del Centro es de 3 Unidades de Infantil, 6 de Primaria, 2 de Eso y 1 de Apoyo a la Integración y una matrícula total de 175 alumnos/as.

Esta particularidad permite que el alumnado de esta pedanía realice el primer ciclo de la ESO en el mismo Centro, sin tener que recurrir al transporte escolar hasta el IES de la localidad más cercana, centro al que está adscrito. Por otro lado, la dotación de profesorado al Centro, específico para el nivel ESO, dota de recursos y personal al mismo y aporta gran ventaja a la hora de su funcionamiento, realización de actividades, proyectos, etc., al mismo tiempo que contribuye a potenciar y elevar el nivel educacional del Colegio.

El edificio escolar ha sufrido sucesivas ampliaciones y reformas en los últimos años, lo que ha contribuido a configurar un Centro escolar disperso, poco funcional y con muchas dependencias adaptadas. Actualmente lo componen cuatro edificios diferenciados (uno dedicado a Administración y dos a Infantil y Primaria). El cuarto edificio lo constituye unas antiguas viviendas de maestros que sucesivamente se han ido adaptando a Aula de Música, Aula de Informática y Biblioteca Escolar. Aunque su estado de conservación es bueno, adolece por la particularidad antes dicha, de falta de espacios: tutorías, despachos, salón de actos, aulas de apoyo y refuerzo, psicomotricidad, etc...lo que obliga en ocasiones a hacer doble o triple uso de una misma dependencia (el laboratorio es a su vez sala de proyección, aula de apoyo y sala de tecnología).

Al mismo tiempo, las instalaciones específicas del Centro, y sobre todo de la parte antigua, tienen graves deficiencias. Instalaciones eléctricas y de calefacción, los cuadros de acometidas son obsoletos, al igual que los servicios de alumno/as y las instalaciones singulares de deportes; no existe porche cubierto. 
Todo ello a pesar del esfuerzo realizado en los tres últimos cursos escolares, en los cuales los edificios del Centro han sufrido una gran reestructuración y reforma para racionalizar su uso y dotarlo de una mejor funcionalidad. Los tres últimos cursos han sido objeto de trabajo en este sentido, mediante la realización de un Plan de Mejora Global, subvencionado en parte por la Junta de Andalucía que ha supuesto una radical transformación del Centro en todos sus aspectos y lo ha preparado para la consecución de los objetivos propuestos.

Uno de los objetivos que se pretende es conseguir la declaración como Centro TIC, así como la concesión del Plan de Apertura - Aula Matinal y Actividades Extraescolares - (Aparicio, 2018). Ambos programas van a suponer una reforma total de las instalaciones obsoletas a través de la Junta de Andalucía, así como la dotación de recursos y herramientas, para de esta forma ofrecer una enseñanza más completa y de mayor calidad para el alumnado.

Todas estas circunstancias y la propia dinámica de los últimos cursos escolares, ha dado como resultado un Centro Escolar, falto de contenidos, recursos humanos y materiales, sin estructura organizativa y funcional y sin los mínimos elementos protocolizados que fija la normativa, valgan como ejemplos el no contar con un plan de autoprotección escolar, protocolo de resolución de conflictos de convivencia, presupuesto económico real, Plan de actividades escolares, etc.

Antes de definir la línea de actuación que se propone, es necesario especificar las características más destacadas del Centro en que nos proponemos actuar:

- El Centro escolar es un centro de educación Infantil y Primaria, de una sola línea, con las que coexisten dos cursos del Primer Nivel de Secundaria. Esto conlleva grandes problemas de horario, material, coordinación y dotaciones.

- Por la anterior circunstancia, la dotación económica que percibe el Centro es muy reducida; la justa para hacer frente a los gastos fijos. Las posibilidades económicas son nulas.

- El Centro se ubica en un entorno reducido -la población total no supera los mil habitantes-, de carácter eminentemente agrícola y con poca dotación social, cultural, etc. y reducidas 
posibilidades en estos campos. Además, es pedanía de un pueblo, de unos tres mil habitantes, donde se reproduce el mismo esquema descrito anteriormente.

- El Ayuntamiento local, por las características descritas, cuenta con un presupuesto municipal reducido, insuficiente para la prestación de los servicios a los que está obligado. Esta carencia presupuestaria incide negativamente en el Centro, y aunque el Ayuntamiento muestra buena disposición, la insuficiencia de medios económicos impide llevar a cabo muchas de sus actuaciones.

- La jornada escolar del Centro es de cinco mañanas, lo que dificulta la organización y coordinación del Centro, motivado por el solapamiento de horarios entre niveles y profesorado.

- Hay graves necesidades de espacios y de materiales. Las aulas de secundaria están habilitadas en antiguas viviendas de profesores, no existe sala de profesores al ser utilizado su espacio por el aula de informática, no hay sala de reuniones niaula de música; se adolecemos de almacenes de material...y un largo etcétera que impiden o dificultan mucho nuestra labor docente. Falta mobiliario adecuado para Secundaria, faltan ordenadores suficientes para la gestión del Centro, la conexión a Internet es una línea básica.

- Hay carencias graves en materia de personal: no hay Portero Escolar, ni Monitor de Infantil ni Auxiliar Administrativo. Existe un logopeda a tiempo compartido con el Centro- del pueblo al que pertenece administrativamente- que es insuficiente para el número de alumnos con problemas de este tipo. El personal de Equipo de Orientación se demuestra insuficiente dado el número de Centros a los que debe atender. Se dispone de una sola limpiadora municipal para los cuatro edificios que configuran el Centro, por lo que la limpieza del mismo no es la adecuada.

- La plantilla docente del Centro no es estable, y tiene poca continuidad. Las plazas vacantes no se ofertan en los concursos de traslados, por lo que son ocupadas continuamente por profesorado de carácter interino, con lo que no se garantiza la 
continuidad (Konieczny, 2015). Consecuencia que hace que nos encontremos en la situación en la que estamos. Se considera en dicho informe que, una plantilla estable planifica a largo plazo, se adapta al Centro, elabora estrategias para próximos cursos y permite una mejor coordinación docente, además de un cambio de actitud de padres y alumnos.

- En los últimos años no ha funcionado la AMPA, a pesar de estar constituida. No ha cobrado cuotas ni ha generado ingresos, por lo que su actividad ha sido nula. En este sentido, la colaboración del sector padres/madres con el Centro ha sido de carácter simbólico (Barrantes, Beltrán \& Pérez, 2016).

A finales del curso, y culminándose este proceso de autoevaluación, se producen una serie de circunstancias que van a condicionar en mucho la marcha global y futura del Centro, y entre las que podríamos citar las siguientes: **a jubilación de la persona que durante los últimos años ha ejercido la función directiva en el Centro. ${ }^{* *}$ El traslado a otro Centro de la persona que ocupaba la Dirección en funciones del Colegio en los últimos meses del curso escolar, así como la función de Coordinación del Plan de Autoevaluación y Mejora del Centro. **Una considerable disminución de la plantilla docente estable en el Centro, por resolución definitiva del concurso de traslados, quedando solo cinco profesores/ as propietarios/as definitivos/as, de una plantilla total de diecisiete profesores/as. **La renovación total de la Junta Directiva de la AMPA del Centro, incorporándose a la misma un grupo de padres y madres nuevos en esta tarea, pero con deseos de trabajar por el Centro. ${ }^{*} \mathrm{El}$ nombramiento por designación directa de nuevo Director del Centro por no haber candidatos a ocupar el cargo entre el profesorado que continuaba en el mismo. Responsabilidad que se designa en el autor de este trabajo. ${ }^{*}$ El nombramiento del nuevo Equipo Directivo del Centro, sin experiencia acumulada. ${ }^{* \star}$ Numerosas bajas en el Consejo Escolar por traslados, dimisión, abandono o pérdida de la condición de miembro al abandonar el Centro. En la actualidad, ninguno de los sectores que integran el Consejo está completo en su totalidad. ** La jubilación de la persona que venía ejerciendo la función de Portero del Centro y coincidiendo con el final del curso escolar.

Todas estas circunstancias vinieron a complicar, y desvirtuar en parte, modificar y agravar el diagnóstico elaborado en el documento del Plan 
de Autoevaluación y Mejora citado anteriormente, así como complicaba y dificultaba su continuidad para el presente curso escolar.

En los últimos meses del curso escolar, se procede por parte del nuevo director, a una reunión con el grupo de profesores/as que continuarían en el Centro, y ante la perspectiva de la casuística apuntada anteriormente, elaboramos un documento titulado "Apuntes para un proyecto", en el que se recogían las bases y los puntos a desarrollar para el ejercicio de una función directiva amparada en una gestión distribuida o compartida, colegiada, colectiva, en definitiva, un "Liderazgo Distributivo", de modo que la iniciativa e influencia esté distribuida entre todos los miembros presente del centro educativo, y de los que se deben incorporar, como medio imprescindible para promover la participación e implicación de todo el profesorado.

Dicho documento parte del conocimiento de la realidad del Centro que, viene dado por los años de permanencia en el mismo y por los resultados que ya ofrecían los cuestionarios realizados por el Plan de Autoevaluación y Mejora, que como ya se ha dicho, estaba en su fase final.

\section{Estrategia innovadora de gestión en el Centro Educativo}

\section{Antecedentes}

Durante el último curso escolar se llevó a cabo en el Centro, la elaboración de una propuesta de Plan de Autoevaluación y Mejora denominado "Observa, piensa, cuestiona y actúa" y que perseguían los siguientes objetivos:

- Analizar de una manera sistemática el contexto y entorno.

- Recibir información, retro-alimentación sobre los trabajos docentes por parte de otros sectores: alumnos, padres, ayuntamiento.

- Contabilizar y jerarquizar los déficit, desde todas las perspectivas: Espacio físico y material, horario general, dotaciones, colaboraciones, ayudas. 
- Sacar conclusiones y perspectivas que faciliten la labor de los docentes.

- Hacer llegar a otros colectivos las esperanzas, inquietudes, quejas e inconvenientes.

- Seguir intentando realizar las finalidades educativas del centro. Sobre todo el punto de comunicación y relación con los distintos sectores. Convivencia y democracia.

- Desarrollar un número mayor de valores transversales y procesos de empatía con la comunidad.

- Mejorar la labor docente y aprovechar mejor los recursos.

- Intentar mejorar la dotación para el funcionamiento del centro.

Para ello, se constituyó el Grupo de Evaluación y Mejora que estuvo integrado por la totalidad de la plantilla docente. También se confeccionó el planteamiento inicial de funcionamiento y el calendario de trabajo a seguir durante el resto del curso escolar para poder concluir el mismo con la elaboración de unas conclusiones o diagnóstico que posibilitase una continuación del plan con una propuesta concreta de intervención.

Durante todo el curso escolar se llevó a cabo el calendario de trabajo establecido y con la participación de los sectores que estaba previsto que interviniesen: profesorado de las tres etapas con las que cuenta el Centro: Infantil, Primaria y Secundaria; los padres y madres del Centro a través de su participación personal, a través de la Asociación de Padres y la participación de los mismos en los órganos colegiados de gobierno del Centro; los alumnos y alumnas a través de los Delegados de clase, participaciones individuales o de grupo y su participación en los órganos colegiados de gobierno; y Ayuntamiento local a través de la Concejalía de relaciones con el Centro así como a través de los propios servicios sociales municipales.

Fruto de ese proceso y trabajo fue el documento "Fase de Diagnóstico" del Programa de Autoevaluación y Mejora del Centro, en el que se recogían los resultados y aportaciones a los cuestionarios cumplimentados por parte de los padres y madres, profesorado y alumnado, con las 
aportaciones presentadas por cada sector y el documento de diagnóstico en el que, se recogían los déficits, carencias, conclusiones y necesidades en todos los aspectos de los que adolece este Centro escolar.

\section{Finalidad de la fase de diagnóstico}

El documento "Apuntes para un proyecto" se fundamentaba en la necesidad de constituir un Equipo Docente que asumiese el proyecto de Centro que se preconizaba, sus principios organizativos y que trabajasen por la consecución de los objetivos que en él se reflejasen; un Proyecto de Centro escolar, realista pero ambicioso, que fuese consensuado por todos los sectores de la comunidad educativa: profesores, padres y administración; el apoyo del sector padres/madres, canalizado a través de una AMPA sólida, activa y participativa; el apoyo institucional, centralizado en el apoyo municipal y por último, un presupuesto económico suficiente que posibilitase la consecución de los objetivos marcados.

Dicho documento, fue reafirmado por todo el profesorado que continuaba en el Centro en siguiente curso escolar, fue entregado a la nueva Junta Directiva de la AMPA, a algunos miembros del Consejo Escolar y se dio a conocer a la Administración local. Todos estos sectores apoyaron mayoritariamente las ideas y objetivos que se contemplaban en dicho documento.

Tras la designación directa al autor del presente trabajo como nuevo Director, a finales del curso pasado, se procedió a la constitución del correspondiente Equipo Directivo. Con el cual, lleve a cabo una estrategia innovadora del Centro fundamentado en las ideas recogidas en este documento citado, de forma que, constituya el cuerpo central del Proyecto de Actuación del Plan de Autoevaluación y Mejora del Centro y al mismo tiempo, hacerlo coincidir con el periodo para el que fue designado, es decir tres años.

Todos los objetivos a conseguir se plantean en la presente estrategia innovadora para los siguientes tres cursos escolares, se basan en los resultados obtenidos en el trabajo realizado durante el anterior curso escolar por el Proyecto de Autoevaluación y Mejora así como por la experiencia y el conocimiento de la realidad del Centro acumulados durante los años de permanencia en el mismo. Muchos de los objetivos a conseguir han sido también propuestos por el sector padres/madres, canalizados a través de la AMPA del Centro. 
Todos los miembros del Equipo Directivo, son conscientes de que la consecución de todos los objetivos que se plantean en el presente Proyecto no va a terminar con la problemática total del Centro. Hay aspectos que escapan a las posibilidades del presente trabajo-como la poca estabilidad o continuidad de la plantilla docente, fenómenos sociales generalizados de cambios de valores, etc.-, pero si, existe en el presente proyecto un convencimiento de que, quizás la actuación innovadora que se pretende, pueda contribuir a mejorar en algo o en mucho el Centro.

Se es consciente también de que, en el presente Proyecto, se hace mucho hincapié en los aspectos materiales y organizativos, pero ello viene dado por la gran necesidad de abordar de una manera seria y eficaz estos aspectos, muy abandonados en el Centro durante muchos años e impuestos por ser un Centro de poca dotación y pocos recursos económicos. Por otro lado se entiende que, abordar definitivamente los aspectos materiales y organizativos posibilita eficazmente la calidad de la enseñanza que se pueda impartir, tal y como se ha justificado a lo largo de este trabajo.

Con todo ello, y desde la autonomía de centro que la ley permite, se espera desde el equipo directivo, que en sucesivos cursos escolares se pueda ir elaborando y abordando estrategias que permitan una mejor coordinación docente, además de un cambio de actitud de padres y madres y alumnos/as, que redunden en la obtención de un Centro de calidad y de mejora social.

El Plan Estratégico que se presenta, está basado principalmente en el diagnóstico efectuado, en el cual, quedo perfectamente recogido los déficits y carencias en todos los aspectos, de los que adolece el Centro escolar.

\section{Objetivos}

Posiblemente el presente Plan Estratégico que se presenta, pueda parecer amplio, pero es concreto. Pueda parecer ambicioso, pero es real. Pueda parecer inalcanzable, pero es viable. El equipo directivo se ve movido por un solo deseo, el de hacer mejor el Centro.

1.- Con relación a los recursos humanos y materiales.

1.1.- Con relación a los recursos humanos. 
1.1.1.- Trabajar por conseguir de la Administración educativa o local la dotación de un Auxiliar Administrativo para Secretaría.

1.1.2.- Trabajar para conseguir un logopeda con horario completo.

1.1.3.- Conseguir mayor dedicación al Centro del personal de equipo de orientación, psicólogo, médico, etc.

1.1.4.- Trabajar por la consecución del monitor de Infantil.

1.1.5.- Trabajar por la consecución de una plantilla estable en el Centro. Pues considero que, una plantilla estable planifica a largo plazo, se adapta al Centro, elabora estrategias para próximos cursos y permite una mejor coordinación docente, además de un cambio de actitud de padres y alumnos.

1.2.- Con relación a los recursos materiales.-

1.2.1- Elaboración de un presupuesto económico del Centro escolar que permita cubrir ampliamente sus necesidades materiales. Para ello se intentará la consecución de más recursos económicos a través de la APA, Ayuntamiento, empresas y particulares, realización de actividades, desarrollo de programas y cursos, venta de productos, subvenciones, etc. Adecuación del gasto a la priorización de necesidades. Presupuesto flexible.

-- Dotación de presupuesto propio para los gastos de funcionamiento y de reposición de material.

-- Realización de la página Web del Centro.

-- Dotación de un ordenador de gran capacidad que actúe como servidor de la Intranet del Centro.

-- Elaboración de la programación del Aula por cursos y niveles.

-- Continuar realizando el compendio de actividades en el entorno TIC, por ser una eficaz herramienta para el uso del Aula y aplicación de la informática a la educación.

-- Realizar inventario de programas educativos.

-- Dotar a cada Aula con, al menos, un ordenador adecuado para soportar una conexión a Internet.

1.3.- Con respecto a la total informatización del Centro.

Se trabajará en: 
-- Ampliación y sustitución de los ordenadores de los que dispone cada Aula.

-- Establecimiento de la Intranet del Centro, realizado los puntos de conexión necesarios en cada edificio o dependencia.

-- Llevar Internet a todos los puntos del Centro.

1.4.- Con respecto al entorno global del Centro y sus dependencias. Se trabajará en:

-- Realización de murales en paredes del patio, que contribuyan a un exorno del mismo.

-- Instalación de bancos en patio de recreo.

-- Delimitación de la zona de Infantil.

-- Decoración de exteriores con macetas y plantas.

-- Montaje de mástiles para banderas en zona de entrada.

-- Instalación de papeleras en patio de recreo y zona de entrada.

-- Colocación de un timbre de entradas y salidas dotado de temporizador.

1.5.- Con respecto al material deportivo.

Se elaborará una relación de necesidades conjuntamente con el profesorado especialista y se trabajará en su consecución y renovación periódica. Se dotará a este Área de presupuesto propio.

1.6.- Conseguir la colaboración de empresas y particulares en el desarrollo del Plan de Mejora.

2.- Con relación a los aspectos organizativos y funcionales.

2.1.- Solicitud, del proyecto de Apertura de Centros de la Consejería de Educación de la Junta de Andalucía.

2.2.- Funcionamiento de escuelas deportivas, de forma reglada.

2.3.- Cursos de Informática en el Aula de Informática a través de Ayuntamiento o APA, como medio de uso del Aula y fuente de recursos económicos.

2.4.- Distribución de las horas del equipo directivo entre el personal implicado en la gestión del Centro. 
2.5.- Establecimiento de un Plan de Excursiones escolares permanente, que posibilite su organización y disponibilidad de materiales escolares.

2.6.- Estudio de la posibilidad de establecimiento de Talleres ínter ciclos.

2.7.- Estudio de la posibilidad de realización de actividades o jornadas únicas a nivel de Centro: día de la limpieza, día del material reciclable.

2.8.- Recopilación de materiales sobre las distintas jornadas que se celebran en el Centro: Navidad, Día de la Paz, Día de la Mujer Trabajadora, Carnaval, Semana Santa, etc., que posibiliten su celebración en sucesivas ediciones.

2.9.- Revisión del ROF y establecimiento de la normativa de funcionamiento. Adecuada publicidad de la misma entre la comunidad educativa para poder exigir su cumplimiento.

2.10.- Revisión de las Finalidades Educativas del Centro.

3.- Con relación al entorno del Centro.

3.1.- Establecer lazos de relación y de colaboración con el Centro del pueblo al cual pertenecemos y con IES del mismo pueblo al cual estamos adscrito, mediante el desarrollo de actividades comunes, visitas, intercambio de experiencias, etc.

3.2.- Apertura del Centro escolar a su entorno con realización visitas escolares, charlas de profesionales, colaboraciones externas, etc.

3.3.- Fomentar y potenciar el funcionamiento de la Asociación de Padres y Madres del Centro.

3.4.- Apertura del Centro escolar mediante el proyecto de Apertura de la Junta de Andalucía. Funcionamiento de Cursos, Talleres, Guardería y escuelas deportivas.

4.- Con relación a los aspectos pedagógicos.

4.1.- Poner en marcha las reuniones de Ciclo, con la temporalización que se considere necesaria. 
4.2.- Establecimiento y reforzamiento de la figura de Delegado/a de Curso.

4.3.- Establecimiento de criterios y actividades comunes a todos los cursos y niveles: recogida de papel para reciclado, asambleas de clase, uso de las nuevas tecnologías, etc.

4.4.- Instauración progresiva del uso de las nuevas tecnologías en la educación en todo el Centro.

4.5.- Participación en convocatorias, planes o programas que convoque la Junta de Andalucía a través de la Consejería de Educación o cualquier otro organismo.

4.6.-Fomento del uso de los recursos y posibilidades que ofrece el Centro de Profesores de la Comarca.

\section{Elaboración}

Tal y como se ha explicado en anteriores apartados, todas las circunstancias y la propia dinámica de los últimos cursos escolares, ha dado como resultado un Centro Escolar, falto de contenidos, recursos humanos y materiales, sin estructura organizativa y funcional y sin los mínimos elementos protocolizados que fija la normativa: valgan como ejemplos el no contar con un plan de autoprotección escolar, protocolo de resolución de conflictos de convivencia, presupuesto económico real, Plan de actividades escolares, etc.

Todo ello, ha llevado a la necesidad de abordar un Plan de Mejora amplio y ambicioso, que aborde no solo un aspecto localizado del Centro, sino que los aborde todos, mediante la realización de las estrategias compartidas o distribuidas necesarias, la adecuada temporalización y la consecución de los suficientes recursos económicos y el apoyo de todos los sectores de la comunidad educativa.

La presente estrategia de innovación se lleva a cabo de una manera flexible, pero coordinada por todos desde el claustro. Entendiendo esta flexibilidad, desde una planificación que nos permita actuar como una hoja de ruta o guía orientativa, más que como una lista de control que, haya que cumplimentar. 
En el caso de las iniciativas que parten del equipo directivo, éste las presenta sucesivamente a los departamentos, el claustro y, en su caso, el consejo escolar.

Con respectoalasiniciativaspromovidasporlos distintos departamentos, el equipo directivo es el encargado de reunirlas, estudiarlas y de proponer un procedimiento para que sean debatidas por sus miembros, el claustro o el consejo escolar. En todo caso, existe por parte del equipo directivo la opinión unánime de que las diferentes iniciativas gocen de bastante autonomía de manera que puedan ajustar las propuestas a las necesidades planteadas. En este sentido, el papel del equipo directivo es clave para mantener su acción más de animadores, proveedores de información, de recursos, consejeros, etc., que de supervisores. En definitiva, lo que se espera es que los distintos departamentos, compartan los recursos creados entre ellos, como materiales o métodos, casi listos para ser usados por todos los demás compañeros. Esto propicia un intercambio continuo de materiales pedagógicos y puesta en marcha que beneficiara al perfecto funcionamiento y objetivo del Centro.

El compromiso y la responsabilidad de todo el profesorado, incluida la comunidad, debe ser la de contribuir activamente todos sus miembros a dinamizar, apoyar y animar, como arma fundamental para que el centro supere los retos y dificultades a los que tiene que enfrentarse. Con ésta estrategia, se presenta un liderazgo que emergerá, desde un grupo de personas que trabajan juntas en torno a unos objetivos comunes.

\section{Temporalización de la actuación}

La temporalización de estas metas y objetivos se fija para los siguientes tres cursos escolares, periodo de duración del mandato del actual Equipo Directivo.

Se estructurará la consecución de las metas y objetivos con carácter trimestral y anual. En determinados objetivos, se concretara las fechas de inicio y fin; en otros casos, se establecerá solamente el inicio, dado que el objetivo tiene carácter general. Se establecerá también, en algunos casos, fechas concretas para cada la consecución de cada una de las fases en que se divida el objetivo final, como es el caso de la confección y puesta en marcha del Plan de Autoprotección Escolar del Centro. 
Para cada curso escolar, se solicitará la continuidad del presente Proyecto, previa aprobación del Equipo de Mejora, Claustro de Profesores y Consejo Escolar del Centro.

\section{Recursos necesarios}

Este Plan Estratégico de Mejora Global que se presenta para el Centro, debe de ir acompañado de los siguientes recursos:

\section{Económicos}

- Los del propio Centro a través de las transferencias corrientes para gastos de funcionamiento del Centro.

- Los que proporcione el propio Proyecto de Mejora, a través de las transferencias para gastos de funcionamiento que el propio Proyecto consiga.

- Los que a este efecto pueda proporcionar la AMPA del Centro o bien la consecución de algunos objetivos que la misma pueda desarrollar.

- Los que pueda proporcionar el Ayuntamiento local o bien la consecución de algunos objetivos que el mismo pueda desarrollar.

- Los que aporten empresas y particulares.

- Los que se generen en el desarrollo de determinadas actividades: carnaval, venta de productos, rifas, etc.

\section{De colaboración}

Se necesitará la colaboración de:

- Claustro de Profesores del Centro.

- Asociación de Padres y Madres del Centro.

- Ayuntamiento local.

- Consejo Escolar del Centro.

- Centro de Profesores de la Comarca.

- Empresas y particulares. 
Lógicamente el presente Plan Estratégico contempla también un plan de formación a todos los niveles de manera que permita el desarrollo eficaz del Plana.

Las demandas en este campo irán surgiendo conforme se vaya desarrollando el Proyecto, pero ya, a priori, es necesario plantear el realizar acciones formativas en relación con la implantación de las nuevas tecnologías. A este respecto, es necesario la celebración de cursos sobre: Curso básico de Informática. Redes. Internet y sus aplicaciones educativas. Escuela de Padres y Madres.

\section{Evaluación del proyecto respecto a la consecución de objetivos, metas e intervenciones.}

El Equipo Directivo realizará la revisión del proyecto con carácter trimestral, comprobando la consecución de los objetivos marcados en ese periodo y la marcha de aquellos que se hayan implantado. El Equipo Directivo podrá, en estas revisiones, alterar el calendario establecido a tenor de las circunstancias que concurran en el Centro y en la marcha del mismo.

A final de cada curso escolar, se realizará la revisión anual, elaborándose una Memoria que será aprobada por el Equipo Directivo, Claustro de Profesores y Consejo Escolar del Centro.

Dicha Memoria servirá de base para establecer las metas y objetivos de la actuación en el siguiente curso escolar y de su calendario de trabajo.

\section{Mecanismos de evaluación por medio de un seguimiento de los indicadores de logro.}

El documento de Autoevaluación y Memoria es el instrumento que se propone. Consta de tres partes fundamentales:

\section{Las fichas de autoevaluación, constituye el núcleo del instrumento evaluativo.}

Pueden ser utilizadas por todos los órganos del centro; no obstante, se propone que esta evaluación sea realizada por el Equipo Directivo o algún órgano o equipo más que se considere oportuno. 
Las fichas de autoevaluación, la valoración de los "indicadores" se hará en una escala de 1 a 4 , siendo este dígito el que señala la valoración más positiva. La casilla "observaciones al indicador" puede servir para aclarar en algún sentido la valoración. Las "fuentes y/o instrumentos de evaluación", se refieren a las fuentes donde es posible obtener mejor información sobre el criterio o algunos de sus indicadores o bien o a los instrumentos por medio de los cuales podemos conseguir esos datos.

\section{El documento síntesis que, junto a las fichas anteriores, conforman la evaluación es la Memoria.}

La memoria debe cumplir tres funciones básicas:

1. Recoger la evaluación interna del centro sobre el Proyecto.

2. Dar un juicio de valor sobre lo realizado, comparándolo con lo previsto y planificado y haciendo explícitos las disfunciones y logros más significativos.

3. Hacer propuestas de mejora referidas a las principales conclusiones extraídas de la valoración de los distintos criterios.

\section{Conclusiones}

La dirección escolar en España, está en un proceso de profunda transformación, para poder satisfacer las necesidades que la sociedad demanda al sistema educativo.

Un proceso que ha ido oscilando desde, un modelo directivo burocrático-administrativo, para pasar a un liderazgo transformador de dirección pedagógica para el aprendizaje, como un factor de primer orden en los logros académicos de los alumnos, así como de los resultados del centro escolar. De aquí que, hoy en día, la mejora de los resultados en un centro escolar se está convirtiendo en una prioridad permanente dentro del sistema educativo, llegándose a relacionar el éxito o fracaso del centro a la dirección de él.

El presente trabajo, ha querido resaltar la importancia del liderazgo educativo, como el camino por el que se debe de avanzar para conseguir 
esa escuela pública de calidad, tal como demanda la sociedad actualmente. Pero también, se ha pretendido dejar claro en este trabajo que, la responsabilidad de esa calidad educativa que se demanda, dependerá en gran medida del propio centro, el cual, debe de estar cargado de cierta cota de autonomía que, permita a cada centro, poder desarrollar sus propios proyectos, adecuándolos a sus contextos. Un contexto a nivel externo, que viene enmarcado en un primer lugar por el contexto socio-económico y cultural en el que se encuentra el centro y, lógicamente también por todos los factores que condicionan el hacer profesional de la dirección (política educativa, normativa reguladora, autonomía, recursos, tipo de escuela...); y un nivel interno que condiciona al líder directivo en el centro escolar, como el profesorado y los órganos de gobierno, factores que se deberán de tener en cuenta a la hora poder pedir responsabilidades al liderazgo directivo de los resultados obtenidos.

La distribución del liderazgo entre diferentes personas y en estructuras organizacionales puede ser una de las maneras de superar las dificultades de las escuelas contemporáneas y de mejorar su eficacia. Éstas pueden fortalecer la administración y la planificación de la sucesión; pues alentar la distribución del liderazgo es una manera de reforzar la planificación de la sucesión y la administración del centro. Para ello, se ha de permitir al personal docente y otro personal del centro participar en el liderazgo, con lo que se ayuda a desarrollar habilidades de liderazgo en el personal y a forjar futuros directores.

Con base enlas características desarrolladas anteriormente, el presente trabajo ha propuesto, como estratégica de liderazgo directivo, un liderazgo distributivo o compartido, como medio y marco que permita satisfacer las necesidades educativas de los centros escolares que, se ven afectado principalmente por una plantilla inestable, con el valor añadido que, todo esto conlleva, a la hora de formar un equipo directivo que, se vea respaldado por los demás órganos de gobierno y que, a su vez, lógicamente permita llevar a buen término el Proyecto Educativo de Centro.

De aquí que, el autor de este trabajo, deje dentro de sus conclusiones la necesidad de que, los directivos de los centros escolares, tengan amplias capacidades también en la gestión del personal, con el fin de contar con el profesorado más adecuado para el proyecto educativo que defiende el centro; es decir, que la autonomía del centro, le permita en parte elegir a sus colaboradores (por ejemplo, a los interinos), de manera que, pueda adaptar los recursos humanos a las necesidades del centro. 
Autonomía que, le permita poder participar en la posibilidad de traslados y reagrupaciones de profesores si así lo piden las necesidades educativas. Como ha quedado recogido últimamente en el Anteproyecto de Ley Orgánica para la Mejora de la Calidad Educativa (LOMCE); que el ministro José Ignacio Wert presentó el pasado día 21 de septiembre de 2012, al Consejo de Ministros, para su posterior debate en el Congreso de los Diputados. Pues, es a través del compromiso de los directivos, los cuales acompañados de la sinergia de todos los miembros de la comunidad educativa, como se conseguirán escuelas de más calidad y más equitativas.

Cualquier reflexión o propuesta que se realice sobre el Liderazgo Directivo Educativo, ha de tener presente y en cuenta una cuestión tan simple sobre qué Escuela queremos, en función de esa cuestión, el sistema educativo deberá marcar las pautas del camino a recorrer, que deben llevarnos a su éxito.

Por eso, el presente trabajo finaliza con la idea principal de que, promover una dirección educativa en los centros públicos en la actualidad $y$, dentro del contexto socio-económico que nos acompaña, y que a su vez permita a esa dirección desarrollar un liderazgo pedagógico, distribuido, estratégico y de sostenibilidad, requiere dotar de atribuciones autónomas necesarias a esa dirección, para que desde el contexto real del centro, permita desarrollar los proyectos educativos y estratégicos que conlleven al objetivo principal: es el aprendizaje y la formación de los alumnos.

\section{Referencias bibliográficas}

Aparicio, O.Y. (2018). Las TIC como herramientas cognitivas. Revista Interamericana de Investigación, Educación y Pedagogía, RIIEP, 11(1). https://orcid.org/0000-0003-35356288

Barrantes, H. A., Beltrán, J. E. P., \& Pérez, F. A. R. (2016). Perfil del estudiante de pregrado de la Facultad de Estudios a Distancia de la Universidad Militar Nueva Granada. Revista Interamericana de Investigación, Educación y Pedagogía, RIIEP, 9(2). DOI: https://doi.org/10.22490/25391887.1948

Bolivar, A., (2010) ¿Cómo un liderazgo pedagógico y distribuido mejora los logros académicos? Revisión de la investigación y propuesta. Magis, Revista Internacional de Investigación en Educación, 3 (5), 79-106. 
de la Calle, C. V., Malaver, M. O., Gallego, J. D. M., Rodríguez, M., Flórez, J. C., Henao, C. E. \& Saldaña, R. (2014). Aportes de los doctorados de educación en ciencia, tecnología y sociedad, desde la sistematización de sus investigaciones doctorales científicas y formativas, 2000-2010. Revista Interamericana de Investigación, Educación y Pedagogía, RIIEP, 7(1). DOI: https://doi.org/10.15332/s1657-107X.2014.0001.04

Díaz, M. J. F. (2007). La dirección escolar ante los retos del siglo XXI. Revista Educativa, 23.

Ferreyra, H. A. (2014). Mesas Socioeducativas para la Inclusión y la Igualdad. Un programa "De todos con todos". Una experiencia en construcción. Revista Interamericana de Investigación, Educación y Pedagogía, RIIEP, 7(2). DOI: https://doi. org/10.15332/s1657-107X.2014.0002.01

Gajardo, M., (2010). Liderazgo distribuido en el campo educativo: pasando del concepto a la práctica. Revista Preal- Formas \& Reformas en la Educación 12 (34).

García, I., (2010). Liderazgo Distribuido una visión innovadora de la dirección escolar: una perspectiva teórica. Revista Omnia, 16 (3) 19-36.

González M., (2003). El liderazgo en tiempos de cambio y reformas. Revista del Fórum Europeo de Administradores de la Educación, 11 (6), 4-8.

Konieczny, P. (2015). Lorenzo García Aretio: bases, mediaciones y futuro de la educación a distancia en la sociedad digital. Revista Interamericana de Investigación, Educación y Pedagogía, RIIEP, 8(1). DOI: https://doi.org/10.15332/s1657107X.2015.0001.08

Langer, E. (2016). La construcción de confianza para el estudio de prácticas de resistencia en la escolarización de jóvenes en contextos de pobreza urbana. Revista Interamericana de Investigación, Educación y Pedagogía, RIIEP, 9(2). DOI: https://doi. org/10.22490/25391887.1945

López, Y. \& Lavié M., (2010). Liderazgo para sostener procesos de innovación en la escuela Profesorado. Revista de curriculum y formación del profesorado, 14 (1).

Martínez J.A., (2010). La dirección escolar, ¿̇una cuestión sin resolver? Tribuna Abierta. CEE Participación Educativa, 5, 74-77.

Pérez, T. H. P. (2013). Aproximaciones al estado de la cuestión de la investigación en educación y derechos humanos. Revista Interamericana de Investigación, Educación y Pedagogía, RIIEP, 6(1). DOI: https://doi.org/10.15332/s1657-107X.2013.0001.05

Pont, B., Nusche, D., \& Moorman, H. (2009). Mejorar el liderazgo escolar, Volumen 1: política y práctica. OECD Publishing. 
Viejo, C. M., Cabezas, I. L., \& Martínez, M. D. J. I. (2013). Las redes de académicas en la docencia universitaria. Revista Interamericana de Investigación, Educación y Pedagogía, RIIEP, 6(2). DOI: https://doi.org/10.15332/s1657-107X.2013.0002.03

Zaitegi, N., (2011) Una nueva dirección para la escuela pública: competencias y transformaciones necesarias. Revista del fórum europeo de administradores de la educación. marzo-abril 2011 (2). 14-18. 